\title{
On seller estimates and buyer returns
}

\author{
Alex Gershkov • Flavio Toxvaerd
}

Received: 4 March 2013 / Accepted: 15 March 2013 / Published online: 30 March 2013

(C) SAET 2013

\begin{abstract}
This paper revisits recent empirical research on buyer credulity in arts auctions and auctions for assets in general. We show that elementary results in auction theory can fully account for some stylized facts on asset returns that have been held to suggest that sellers of assets can exploit buyers by providing biased estimates of asset values. We argue that, rather than showing that buyers are credulous, the existing evidence can serve as an indirect test of the rationality assumptions underlying auction theory.
\end{abstract}

Keywords Auctions · Information disclosure - Seller manipulation · Buyer credulity

JEL Classification $\quad \mathrm{D} 44 \cdot \mathrm{D} 82 \cdot \mathrm{G} 12 \cdot \mathrm{G} 14$

\section{Introduction}

Asymmetry of information is pervasive in markets for financial assets. Sellers of assets such as shares of newly floated companies as well as objects of fine art may have better

\footnotetext{
A. Gershkov

Department of Economics, Faculty of Social Science, Hebrew University of Jerusalem, Mount Scopus, 91905 Jerusalem, Israel

e-mail: alexg@huji.ac.il

A. Gershkov

School of Economics, Faculty of Business,

Economics and Law University of Surrey Guildford, Surrey GU2 7XH, United Kingdom

F. Toxvaerd $(\varangle)$

Faculty of Economics, University of Cambridge and CEPR,

Sidgwick Avenue, Cambridge CB3 9DD, United Kingdom

e-mail: fmot2@cam.ac.uk
} 
information about true values than potential buyers, and have an inherent interest in distorting sales prices upwards. This fact raises the concern that buyers may be lead to overpay for assets and let themselves be fooled by information supplied by the seller. The empirical evidence seems inconclusive. Bradshaw et al. (2003) confidently assert that "[the] evidence [...] suggests that investors are systematically fooled by analyst hype". If such conclusions are taken at face value, we are facing a potentially huge problem of market inefficiency which casts a shadow on the investment advice provided by sellers of assets. It is therefore of great importance to study the robustness and interpretations of studies suggestive of such sell-side manipulation and buyer gullibility.

In recent empirical research, Mei and Moses (2005) describe and study a natural experiment that occurred in the market for fine art. Prior to 1973, buyers of art at auction houses such as Sotheby's and Christie's largely relied on own information and research when valuing pieces put up for sale. After 1973, both auction houses publicly announced valuations of all items offered for sale. This situation provides an ideal context within which to test the effects that announced seller valuations have on prices of assets. Their main findings can be summarized as follows: (i) Auctioneer price estimates are biased, (ii) Bidders tend to pay more for objects with high auctioneer price estimates and (iii) The introduction of auctioneer price estimates caused long-term under performance of auctioned objects. The authors take these findings to suggest that buyers of art are credulous and that auction houses exploit this fact to further their own interests.

In this paper, we revisit these findings by providing a simple formal model of the aforementioned natural experiment, in which all bidders are fully rational. Within this model, we show that it is consistent with equilibrium behavior for bidders to bid more aggressively for items with higher announced valuations and that the shift from a regime without public valuations to one with such valuations can lead to a decrease in long-term returns for bidders.

Mei and Moses' (2005) finding (i), i.e. that auctioneer estimates are biased (significantly so, although the bias is quite small), should be contrasted to previous studies that fail to find the existence of bias, such as Keane and Runkle (1988). McAndrew et al. (2012) argue that the bias found in previous studies stems from the exclusion in the data of assets that do not reach the reserve price (and are thus not traded). They show that once all auctioned objects are considered (sold and unsold alike), there is no evidence of bias. In a related paper, Sproule and Valsan (2006) consider a hedonic model of art auctions and find that it has no better predictive power than those of estimates provided by sellers of the assets.

In itself, estimation bias is not of interest in the current context unless bidders are found to react to announced price estimates. In other words, if bidders were oblivious to announced price estimates, such estimates would be inconsequential. This motivates Mei and Moses' (2005) analysis of bidder reactions to public price estimates. They find that bidders tend to pay more for items with higher announced auctioneer price estimates.

What should or can one conclude based on such an observation? Clearly, were it indeed the case that auction houses and sellers of assets were manipulating information and exploiting gullible bidders by providing them with biased price estimates, such a 


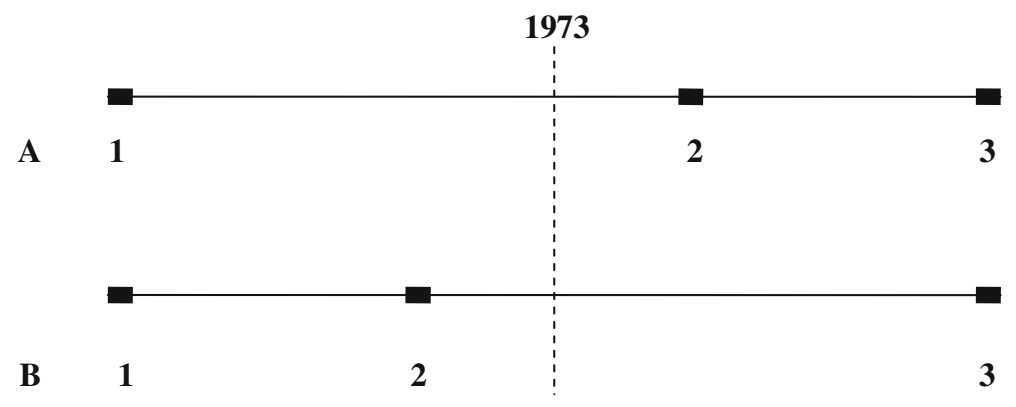

Fig. 1 The two scenarios under the two regimes

finding would be expected. Unfortunately, concluding that bidders are gullible would be premature, since such a pattern in price reactions is equally consistent with a more benign state of affairs, namely that sellers truthfully reveal the information they possess and that bidders in turn rationally bid more aggressively for higher value items. This difficulty leads to the natural experiment provided by the introduction of public price estimates in 1973.

The approach adopted by Mei and Moses (2005) is to consider what effects the regime change had on bidder returns. If the introduction of price estimates changed bidder returns to the worse, they argue, this would constitute prima facie evidence that bidders are being misled by the manipulated information provided by auction houses. Specifically, their analysis is as follows. They consider a large data set of prices of items for which three consecutive sales have been recorded. This allows us to determine the effects (if any) that the introduction of price estimates had on returns to a particular item over different holding periods (i.e. the adjusted change in price from the date at which the item was acquired until the date at which it was resold). Figure 1 shows their setup schematically. For some part of the data set (scenario A), the first sale occurred prior to 1973 while the next two sales occurred after 1973. The remaining part of the data set (scenario B) consists of items which had two recorded sales prior to 1973 and one recorded sale after.

The strategy of their analysis is as follows. If, as the authors conjecture, the introduction of price estimates creates an upward pressure on prices, only items sold in the second regime (i.e. post price estimate introduction) would be thus influenced. In turn, this would mean that the return in holding period 1 (from $t=1$ to $t=2$ ) in scenario A would be larger than the return in holding period 1 in scenario B. Similarly, the return in holding period 2 (from $t=2$ to $t=3$ ) in scenario A should be lower than the return in holding period 2 in scenario $\mathrm{B}$.

Below, we shall argue that both findings (ii) and (iii) in Mei and Moses (2005) are consistent with fully rational behavior and furthermore that both observations are essentially reflections of the same mechanisms at work. As a consequence, their result (i) also becomes irrelevant. Specifically, our setup is as follows. We consider two different regimes, consistent with the situations prevailing before and after the introduction of auctioneer valuations. In the first regime, bidders receive private signals before participating in a standard English auction. No further information is disclosed 
before bidding takes place. In the second regime, everything is as in the first regime, with the difference that before bidding takes place, the auctioneer publicly announces all bidders' signals. We then go on to study two different scenarios, like scenarios A and B in Mei and Moses' (2005) study. We show that in equilibrium, the return in holding period 1 in scenario A is larger than the return in holding period 1 in scenario B. Similarly, we show that the return in holding period 2 in scenario A is lower than the return in holding period 2 in scenario B.

In short, we show that the decrease in long-term return on assets during holding periods that straddle the introduction of publicly announced auctioneer valuations are due to the fact that more information is released, which in turn prompts bidders to rationally bid more aggressively than they otherwise would have.

\section{Model and analysis}

A single object is offered for sale to a set $N=\{1, \ldots, n\}$ of potential risk neutral buyers. The object will be resold several times using an English auction. ${ }^{1}$ Prior to the auction at time $t=1,2,3$, each bidder $i$ privately observes the realization of a signal $s_{i}^{t} \in S_{i}^{t}=[0,1]$. This signal is informative about the object's future resale value and possibly some intrinsic value.

We assume that the value of the object to bidder $i$ is $U_{i}\left(s^{t}\right)$, where $s^{t}=\left(s_{1}^{t}, \ldots, s_{n}^{t}\right)$ is the vector of observed signals of all bidders in period $t$. Denote by $s_{-i}^{t}$ the vector of observed signals of all bidders but $i$. Let $U_{i}\left(s^{t}\right) \equiv U\left(s_{i}^{t}, s_{-i}^{t}\right)$, i.e. the value function is invariant to permutations of its last $n-1$ arguments. Finally, we assume that for all $j \in N$,

$$
\frac{\partial U\left(s_{i}^{t}, s_{-i}^{t}\right)}{\partial s_{i}^{t}} \geq \frac{\partial U\left(s_{i}^{t}, s_{-i}^{t}\right)}{\partial s_{j}^{t}}>0
$$

In short, condition (1) means that a bidder's own signal affects his valuation at least as much as the signals of rival bidders. If the first inequality holds as a strict equality, then we are in the pure common values case. The last inequality excludes the pure private values setting in which the auctioneer's estimate of the object's value is irrelevant for the bidders.

Note that the function $U_{i}\left(s^{t}\right)$ can be thought of as a reduced form for some suitably discounted expected future stream of earnings. The chosen setup allows for both common value and private value components. The possibility of future resale of bought assets generates a common value element, while different tastes in art or different portfolio requirements can account for a private value component.

We consider the following two regimes. In the first regime, no additional information is revealed, while in second regime, the auctioneer announces some message that completely reveals all relevant information to all bidders.

1 This setup is chosen to closely match the natural experiment described above. Other possibilities have been analyzed in the literature. E.g. Virag (2013) studies a first-price auction, which is followed by resale through a second-price auction with a reserve price. 
Figure 1 shows our setup schematically. Before 1973, we are in the first regime while after 1973, we are in the second regime. What we want to determine is what impact the disclosure of information by the auctioneer has on the return on an asset, defined as the difference between the price of an object when acquired by a bidder and the obtained price when the object is later resold. In particular, we will compare the return in holding period 1 (from $t=1$ to $t=2$ ) and in holding period 2 (from $t=2$ to $t=3$ ) in scenario A versus scenario $\mathrm{B}$, consistent with the setup considered by Mei and Moses (2005).

In order to make this comparison, we will first determine the equilibrium of auctions conducted under the two different regimes.

We analyze the situation as a sequence of one shot games and not as a repeated game, since even though we assume that the number of the participants does not change, the set of players may differ from one period to the next. However, this does not preclude the signals from being correlated across periods. What we do exclude is that a given period's signals can be perfectly inferred from knowledge of previous periods' signals.

\subsection{First regime (auction without auctioneer estimate)}

The following reasoning follows that of Milgrom and Weber (1982). In an English auction, initially all bidders are active at the price 0 . As the auctioneer increases the price, bidders drop out one by one and no bidder who has dropped out can become active again. After any bidder quits, all remaining active bidders know the price at which he quit. Therefore, a strategy of a bidder specifies whether, at a given price level $p$, he will remain active or drop out, as a function of his signal, the number of bidders who have quit the bidding, and the levels at which they quit.

Let $k$ denote the number of bidders who have quit and let $p_{1} \leq \cdots \leq p_{k}$ denote the levels at which they did so. Then, bidder $i$ 's strategy can be described by functions $b_{k}\left(s_{i}^{t}, p_{1}, \ldots, p_{k}\right)$ which specify the price at which bidder $i$ will quit if, at that point, $k$ other bidders have quit at the prices $p_{1}, \ldots, p_{k}$.

Denote by $\hat{s}_{j}^{t}$ the $j$ th highest signal among all bidders but $i$. Then, the following strategies constitute a symmetric Bayesian Nash equilibrium: ${ }^{2}$

$$
\begin{aligned}
b_{0}\left(s_{i}^{t}\right)= & U\left(s_{i}^{t}, s_{-i}^{t}\right) \text { where } s_{i}^{t}=s_{j}^{t} \quad \forall i, j \\
b_{k}\left(s_{i}^{t}, p_{1}, \ldots, p_{k}\right)= & U\left(s_{i}^{t}, s_{-i}^{t}\right) \text { where } s_{j}^{t} \\
& = \begin{cases}s: b_{j-1}\left(\hat{s}_{n-j}^{t}, p_{1}, \ldots, p_{j-1}\right)=p_{j} & \text { if } j \text { quit at } p_{j} \\
s_{i}^{t} & \text { otherwise }\end{cases}
\end{aligned}
$$

In words, this strategy means that if nobody dropped out yet, every bidder remains active until the price reaches his expected utility, conditional on the event that all bidders observe exactly the same signal as his own. If some bidders dropped out,

\footnotetext{
2 It can be shown that the following strategies constitute an ex-post equilibrium. For a formal proof, see Milgrom and Weber (1982).
} 
the remaining bidders learn the signals of these bidders. In this case, the equilibrium strategy of the active bidders is to update their valuations, only for the signals of the bidders that decided to quit.

The considered strategies deserve further comment. In principle, the bidders could employ strategies at some auction that condition on previously revealed information. Consider the auction at $t=2$. In this auction, all bidders know the price levels at which players quit at the auction at $t=1$, which in turn reveals their respective valuations at that point in time. But, if signals are correlated over time, this information is informative also about the players' current valuations.

Should they take this information into account when deciding when to quit in the current auction? We argue that it is indeed consistent with equilibrium behavior not to do so. The arguments is as follows. Assume that all bidders but $i$ use the strategies described above. Bidder $i$, even after observing the true realization of the other bidders' signals, would not want to change his strategy because we are in an ex-post equilibrium. Therefore, he would not want to change his strategy based on incomplete information about the other bidders' signals, as provided by knowledge of the signal realizations in previous rounds. ${ }^{3}$

\subsection{Second regime (auction with auctioneer estimate)}

Now assume that before conducting the auction, the auctioneer reports a message that reveals all signals to all bidders. In this case we have a game of complete information, and there is a dominant strategy equilibrium in which every bidder quits once the price reaches his value of the object, regardless of the prices at which other bidders quit. That is, we have

$$
b(s)=U\left(s_{i}^{t}, s_{-i}^{t}\right)
$$

Note that because in this regime we have complete information, these bidding strategies constitute ex-post equilibrium strategies.

\subsection{Comparison of the two regimes}

Let $b_{(i)}^{t}$ be the $i$ th highest bid in period $t$, and let $\sigma$ be the auctioneer's message. With this notation, we can now state the following result:

Theorem 1 Revenues in the second regime are higher than or equal to the revenues in the first regime. Formally,

$$
b_{(2)}^{2}\left(s^{2}, \sigma\right) \geq b_{(2)}^{2}\left(s^{2}\right)
$$

Proof Note that in the first regime, the payment of the winner equals the utility of the second highest bidder, conditional on all signals less than his own and on the event

\footnotetext{
3 It should be noted though that there may be equilibria in which all bidders do take such knowledge into account.
} 
that the highest signal equals his own. In the second regime, the expected payment equals the utility of the second highest bidder, conditional on all signals less then his own and on the true highest signal which is strictly higher than his own signal (with probability 1)

Note that the theorem also implies that expected revenues in the second regime are strictly higher than in the first regime. In fact, the realization of this fact may well have prompted the auction houses to start providing pre-auction price estimates in the first place.

\subsection{Returns on assets across scenarios}

Now consider the returns on an asset, depending on whether or not the holding period straddles the point in time where the auctioneer announces his information to the bidders. Note that we have implicitly assumed that the bidders in the first (pre-announcement) regime do not anticipate the auctioneer's future information disclosure. Denote by $\Delta_{r}^{h}$ the return in holding period $r=1,2$ in scenario $h=A, B$. The relevant returns are then given by

$$
\begin{aligned}
\Delta_{1}^{A}\left(s^{1}, s^{2}, \sigma\right) & \equiv b_{(2)}^{2}\left(s^{2}, \sigma\right)-b_{(2)}^{1}\left(s^{1}\right) \\
\Delta_{2}^{A}\left(s^{1}, s^{2}, \sigma\right) & \equiv b_{(2)}^{3}\left(s^{3}, \sigma\right)-b_{(2)}^{2}\left(s^{2}, \sigma\right) \\
\Delta_{1}^{B}\left(s^{1}, s^{2}\right) & \equiv b_{(2)}^{2}\left(s^{2}\right)-b_{(2)}^{1}\left(s^{1}\right) \\
\Delta_{2}^{B}\left(s^{1}, s^{2}, \sigma\right) & \equiv b_{(2)}^{3}\left(s^{3}, \sigma\right)-b_{(2)}^{2}\left(s^{2}\right)
\end{aligned}
$$

From these definitions and Eq. (5) of Theorem 1, the following results follow:

\section{Theorem 2}

$$
\begin{array}{r}
\Delta_{1}^{A}\left(s^{1}, s^{2}, \sigma\right)-\Delta_{1}^{B}\left(s^{1}, s^{2}\right)=b_{(2)}^{2}\left(s^{2}, \sigma\right)-b_{(2)}^{2}\left(s^{2}\right) \geq 0 \\
\Delta_{2}^{A}\left(s^{1}, s^{2}, \sigma\right)-\Delta_{2}^{B}\left(s^{1}, s^{2}, \sigma\right)=b_{(2)}^{2}\left(s^{2}\right)-b_{(2)}^{2}\left(s^{2}, \sigma\right) \leq 0
\end{array}
$$

In words, (10) means that the return in holding period 1 in scenario $\mathrm{A}$ is higher than the equivalent return in scenario B. Similarly, (11) means that the return in holding period 2 in scenario $\mathrm{A}$ is lower than the equivalent return in scenario $\mathrm{B}$.

These results should be contrasted with the empirical findings from arts auctions summarized above. Indeed, the model we have analyzed can predict exactly the price movements that Mei and Moses (2005) found in the data.

\section{Robustness and extensions}

These results, while derived in a very simple setting, are quite robust. First, as already mentioned, we allow for almost any type of dependence of signals across periods. 
Actually, the equilibria we have derived are distribution free, meaning that none of the strategies or resulting equilibria depend on distributional assumptions.

One may wonder if the results would be changed if the auctioneer, instead of revealing all available information, could somehow garble his signal when informing the bidders. If the auctioneer can commit ex-ante to a disclosure policy, then he would always find it optimal to fully disclose whichever information he has. This is an instance of the linkage principle (see Milgrom and Weber 1982).

What would happen if the auctioneer, instead of having perfect information, only has noisy or imperfect information at his disposal? In that case, our results would continue to hold under the additional assumption that the auctioneer's signal as well as all the bidders' signals are affiliated. Affiliation can be thought of roughly as strong positive correlation.

We should comment on our choice of equilibria. In both regimes, we have picked specific equilibria and then compared these. For completeness, it should be mentioned there exist other equilibria which may yield different results. In the first regime, we have focused only on symmetric equilibria. There may exist other, asymmetric equilibria in which the bidders employ complicated strategies. We disregard these for simplicity.

Next, it should be pointed out that the equilibrium strategies considered under the first regime would also constitute equilibrium strategies under the second regime. In this case, the equilibrium would be in dominated strategies but constitute a Nash equilibrium nevertheless. ${ }^{4}$ We focus on the dominant strategy equilibrium since these are a natural first approach in a complete information game.

As described in the introduction, the fact that equilibrium prices are increasing in the auctioneer's announced valuation can be understood in terms of the aforementioned linkage principle. ${ }^{5}$ Although not derived in the context of the above model, the reasoning can be understood as follows. In a nutshell, the linkage principle states that if an auctioneer is able to credibly commit to any information revelation policy, he can do no better than to truthfully reveal all available information to the bidders prior to the auction. This principle holds for several auction formats such as English, second price, first price and others. In consequence, if the auctioneer's estimate increases, the bidders believe the auctioned object to be of greater value and thus rationally increase their bids. In this way, the linkage principle yields exactly the relationship between estimates and equilibrium prices identified in the data by Mei and Moses (2005). ${ }^{6}$

Last, the English auction format has been assumed in the above analysis for expositional simplicity and because the data analyzed by Mei and Moses (2005) was generated by English auctions. The results would continue to hold for the second price auction as well as for the first price auction under the additional assumption that signals are independent across periods.

\footnotetext{
4 In this equilibrium though, the bidders ignore the auctioneer's message and use the same strategies as in the first regime.

5 See Milgrom and Weber (1982) for a formal statement and proof of this principle.

6 This principle is quite robust, holding for all the formats previously mentioned as long as the auctioneer's signal is affiliated with the bidders' signals.
} 


\section{Discussion}

In this paper, we revisit recent empirical research on auctioneer estimates and their reported adverse effects on bidder returns. We argue that elementary results in auction theory yield predictions consistent with such empirical findings, thus casting doubt on the validity of the claim that buyers are fooled by the information provided by interested sellers. In contrast, we believe that the empirical findings can serve as an indirect test of some of the basic tenets of auction theory, among them the notion that bidders are fully rational and cannot be misled in equilibrium.

The interesting question of buyer credulity remains valid though. The difficulty in showing buyer gullibility is that the signs of the price changes that could potentially show that bidders are misled are, as we have shown, the same as those predicted by a model of fully rational agents. This means that it is insufficient to look at the signs of price changes and that the magnitude of these should be more closely studied. In conclusion, evidence of bidder credulity should be provided by showing that the price reactions to publicly announced auctioneer price estimates are significantly over and above the levels predicted by the assumption of rational behavior. Empirically implementing such a study would be interesting and is left for future research.

Our analysis also carries lessons for markets for other assets than fine art, as nothing in our model is particular to the art market.

Acknowledgments This paper was previously circulated under the title Auctioneer Estimates and Credulous Buyers Revisited. We wish to thank Emanuele Giovannetti and João Amaro de Matos for useful comments on an earlier draft of this paper. We also thank participants at the Meetings of the American Economic Association, Philadelphia, 2005, the Meetings of the European Economic Association, Budapest, 2007 and seminar participants at the Office of Fair Trading, UK.

\section{References}

Bradshaw, M.T., Richardson, S.A., Sloan, R.G.: Pump and Dump: An Empirical Analysis of the Relation Between Financing Activities and Sell-Side Analyst Research. (2003)

McAndrew, C., Smith, J.L., Thompson, R.: The impact of reserve prices on the perceived bias of expert appraisals of fine art. J. Appl. Econom. 27(2), 235-252 (2012)

Keane, M.P., Runkle, D.E.: Are financial analysts' forecasts of corporate profits rational? J. Political Econ. 106(4), 768-805 (1998)

Mei, J., Moses, M.: Vested interest and biased price estimates: evidence from an auction market. J. Finance 60(5), 2409-2435 (2005)

Milgrom, P., Weber, R.: A theory of auctions and competitive bidding. Econometrica 50(5), 1089-1122 (1982)

Sproule, R., Valsan, C.: Hedonic models and pre-auction estimates: abstract art revisited. Econ. Bull. 26(5), $1-10$ (2006)

Virag, G.: First-price auctions with resale: the case of many bidders. Econ. Theory 52(1), 129-163 (2013) 